\title{
Philosophiques
}

\section{Croyance et spontanéité}

\section{Pascal Engel}

Volume 36, numéro 1, printemps 2009

L'idéalisme britannique

URI : https://id.erudit.org/iderudit/038019ar

DOI : https://doi.org/10.7202/038019ar

Aller au sommaire du numéro

Éditeur(s)

Société de philosophie du Québec

ISSN

0316-2923 (imprimé)

1492-1391 (numérique)

Découvrir la revue

Citer ce document

Engel, P. (2009). Croyance et spontanéité. Philosophiques, 36(1), 215-220.

https://doi.org/10.7202/038019ar d'utilisation que vous pouvez consulter en ligne.

https://apropos.erudit.org/fr/usagers/politique-dutilisation/ 


\title{
Croyance et spontanéité
}

\author{
PASCAL ENGEL \\ Université de Genève
}

Dans la conférence III de L'esprit et le monde ( $2^{\mathrm{e}}$ éd., $\mathbb{S}$ 6, p. 60-63, trad. fr. p. 93-96), quand il discute les thèses d'Evans sur le contenu non conceptuel, John McDowell fait quelques remarques sur la croyance et le jugement. Ces remarques sont quelque peu orthogonales par rapport à l'argumentation générale du chapitre, que je n'examinerai pas ici (je l'ai fait ailleurs) ${ }^{1}$, mais elles me semblent à la fois signifcatives pour l'ensemble de son projet dans le livre, en même temps que problématiques.

Evans s'intéresse à la distinction entre le contenu informationnel de l'expérience perceptive et le contenu des croyances, et il rejette l'idée que le premier pourrait constituer des « inclinations prima facie à croire ». Il a l'intention, nous dit McDowell, de réserver le terme "croyance » pour désigner un état cognitif plus sophistiqué, lié à la notion de jugement et à celle de raison. McDowell ajoute:

Il faut réserver l'idée de croyance pour quelque chose qu'on ne peut comprendre que dans le contexte de l'idée de spontanéité, la spontanéité étant l'entreprise active par laquelle un sujet acquiert un contrôle rationnel sur la configuration de sa pensée. Nos croyances ne résultent pas toutes de cette activité d'organisation, mais on a intérêt à réserver le titre de croyance à un état cognitif qui ressortit à cette activité. Même lorsque je n'ai pas décidé d'avoir une certaine croyance, on peut toujours me demander de quel droit j'ai cette croyance. Nous pouvons résumer ces suggestions d'Evans en disant que la croyance est une disposition à émettre des jugements, et que le jugement est essentiellement un acte de spontanéité ( $\mathrm{M} \& \mathrm{~W}, 60$, trad. fr., 94).

McDowell emprunte ici l'idée d'Evans selon laquelle le contenu de la croyance diffère du contenu de l'expérience en ceci que le premier, mais pas le second, implique l'exercice de capacités conceptuelles et une sensibilité à la raison, et il soutient que cette sensibilité et cet exercice sont déjà présents dans l'expérience elle-même. Selon McDowell, cela ne veut pas dire que l'expérience soit par elle-même l'exercice de jugements actifs, car l'expérience, selon lui, est passive. Mais les capacités requises pour avoir l'expérience sont conceptuelles et exploitées dans les jugements. Faisons ici abstraction de la thèse mcdowellienne selon laquelle le contenu de l'expérience est conceptuel, et

1. Mon excuse pour le choix que je fais ici de discuter ce qui semble être une question secondaire par rapport à la ligne générale d'argumentation du livre de Mc Dowell, étant donné que j’ai déjà discuté les vues de Mc Dowell sur la perception et la vérité comme identité de Mc Dowell ailleurs (Engel, 2001, 2002, 2003; voir aussi la réponse de Mc Dowell [2004] à ce dernier article). 
occupons-nous seulement de sa thèse sur la croyance et le jugement avancée dans ce passage. En quel sens pouvons-nous dire que la croyance est "active» et «essentiellement un acte de spontanéité »?

McDowell a défendu ailleurs une conception du jugement proche de celle qu'il affirme dans le passage cité plus haut:

Juger, se faire une opinion pour savoir quoi penser est quelque chose dont nous sommes, en principe, responsables - quelque chose que nous faisons librement, par opposition à quelque chose qui se produit simplement dans nos vies. Bien sûr la croyance n'est pas toujours, ni même ordinairement, le résultat de l'exercice de cette liberté de faire ce que nous pensons. Mais même si une croyance n'est pas librement adoptée, elle est l'actualisation de capacités d'un certain type, les capacités conceptuelles, dont le mode d'actualisation paradigmatique est l'exercice de la liberté en quoi consiste le jugement. Cette liberté, exemplifiée dans des actes responsables de jugement, consiste essentiellement dans le fait d'être capable de répondre à des critiques à la lumière de considérations rationnellement pertinentes. Ainsi, le royaume de la liberté, tout au moins comme royaume de la liberté de juger, peut être identifé à l'espace des raisons $(1998,434)$.

Selon cette conception, on a un contrôle sur ses croyances, et celles-ci sont en ce sens l'exercice d'un pouvoir actif et libre qui nous rend responsables de ce que nous croyons, quand nous avons la capacité d'être sensibles aux raisons. Appelons ce pouvoir contrôle rationnel. McDowell insiste sur le fait que le contrôle actif que nous avons sur nos croyances dans le jugement ne consiste pas en notre capacité à "croire à volonté » ou à "décider de croire ", que ce soit de manière directe ou indirecte. Il ne propose pas une forme de volontarisme doxastique. Mais on peut néanmoins se demander: si notre responsabilité dans le jugement ne vient pas d'un acte de la volonté, d'où vient-elle? Elle vient, selon McDowell, du fait que quand nous formons un jugement "nous sommes capables de prendre le contrôle rationnel de la forme de notre pensée ", et du fait que nous nous trouvons situés dans "l'espace des raisons ». Bien qu'il y ait eu sans aucun doute, depuis que McDowell a repris cette célèbre formule de Sellars, un abus dans l'usage de l'expression - l'espace des raisons —, il n'est pas difficile de reconnaître ici la notion kantienne de la liberté comme autonomie, que d'autres auteurs inspirés par Sellars ont également développée. Par exemple Robert Brandom écrit:

La réconciliation opérée par Kant entre notre nature comme être libres du fait de notre rationalité, en tant que nous sommes rationnels - et par conséquent de la liberté comme contrainte par une certaine sorte de norme, les normes de rationalité — implique que l'on traite le statut normatif de l'obligation morale comme institué par des attitudes normatives (Brandom, 1994, 51)².

Mais il nous faut encore comprendre comment un sujet qui se trouve contraint par les normes de la raison peut être libre dans ses jugements et

2. Voir aussi Burge, 1995, pour une position voisine sur ce point de celle de McDowell. 
responsable de ceux-ci. Si je comprends bien la thèse de McDowell, ici, le type de liberté dont nous disposons dans la croyance répond à au moins deux conditions. Un sujet qui croit quelque chose est responsable de sa croyance quand: i) il est capable d'être sensible aux raisons et aux normes de la croyance; et ii) il est sensible aux raisons s'il est capable de réfléchir sur ses raisons de croire. Associée à la condition (i) suit l'idée que le sujet doit reconnaître son engagement vis à vis d'un type spécifique de normes, les normes de la raison. La condition (ii), cependant, est moins claire. McDowell admet dans le premier passage que toutes nos croyances ne sont pas le résultat de notre "activité de se faire un avis": certaines de nos croyances ne sont pas conscientes ou du moins ne sont pas complètement conscientes. Mais le fait nous puissions, dans certaines circonstances, exercer notre jugement de manière critique est conditionné par le fait que nous soyons réflexivement conscients de nos jugements, et capables de souscrire à des engagements aux normes que nous reconnaissons.

Toutefois, c'est là que je vois une difficulté pour la conception (et pour toute conception de type kantien au sens large). Si nous essayons de spécifier ces conditions, il n'est pas clair qu'il y ait un sens dans lequel nous puissions dire qu'une croyance est "active» ou «le produit de la spontanéité». En premier lieu, est-ce que le fait de se conformer à une norme, y compris une norme de la raison, est le produit d'un acte de l'esprit? Pour qu'on ne considère que les normes logiques, qui, on peut le supposer, forment la base de l'espace des raisons, ces dernières sont habituellement considérées comme des contraintes auxquelles on obéit sur le mode passif (on ne souscrit pas activement à un principe comme le principe de non-contradiction). Même si l'on n'a pas à souscrire à l'injonction d'Achille à la tortue dans la fameuse histoire de Lewis Carroll — «la logique te prendra par la gorge et te forcera à inférer» -, même si l'on ne succombe pas à l'image de la "dureté du "doit" logique ", notre engagement vis-à-vis des normes logiques est tout sauf actif.

En second lieu, la condition (ii) sur le jugement, selon laquelle il doit être réflexif, est problématique. Car si nous supposons qu'un sujet qui suit les normes de la raison doit former un jugement d'ordre supérieur selon lequel il suit les normes de la raison, en quoi ce jugement d'ordre supérieur peut-il avoir le moindre effet sur son jugement de premier ordre? C'est là aussi l'une des leçons de l'histoire de Lewis Carroll: le fait de juger que l'on est soumis à une norme n'est pas suffisant en soi pour mouvoir l'esprit. C'est pourquoi, dans l'histoire d'Achille et de la Tortue le seul fait d'ajouter la proposition « Si, si $\mathrm{P}$ et si $\mathrm{P}$ alors $\mathrm{Q}$, alors $\mathrm{Q}$ » à la liste des propositions ne permet pas d'inférer la conclusion. De même si l'on ajoutait la proposition selon laquelle l'inférence en modus ponens est une norme logique ${ }^{3}$. Le simple fait d'avoir

3. Ce point est bien mis en valeur par Blackburn (1995), qui l'applique au raisonnement pratique. 
un jugement d'ordre supérieur selon lequel une certaine norme est en place ne nous fait pas par elle-même suivre la norme.

Mais une autre option est ouverte au kantien. Il peut soutenir qu'il y a un certain type de contrôle que nous avons sur nos jugements, mais qui n'est pas de nature réflexive. C'est l'idée, qui vient aussi de Gareth Evans, et qui a été reprise par plusieurs auteurs récemment, selon laquelle quand nous formons un jugement relativement à la vérité d'une proposition, nous ne «montons » pas à un jugement réflexif selon lequel ce que nous croyons, P, est vrai, mais nous nous demandons simplement à nous-même si c'est le cas que $P^{4}$. On peut soutenir que la norme de base de la croyance - une croyance est correcte si et seulement si elle est vraie - est implicitement en œuvre dans l'exercice de tels jugements. Certains auteurs, comme Hironymi (2006), parlent dans ce cas du "contrôle évaluatif » que nous avons sur nos croyances et opposent ce type de contrôle au type de contrôle "manipulatif » que nous avons sur nos croyances quand nous avons l'intention de les avoir pour des raisons pratiques et non pas épistémiques. Dans le contrôle évaluatif en ce sens, il y a bien évaluation, mais comment le fait que cette évaluation soit constitutive de la relation immédiate que nous avons avec nos croyances peut-il constituer une forme de contrôle sur elles? Juger si P n'est pas contrôler son attitude vis-à-vis de $\mathrm{P}$. Au mieux cela peut être une précondition du fait d'avoir d'autres attitudes, ou d'être capables de réviser ses croyances à la lumière de nouvelles informations, ou pour s'engager dans un processus d'enquête. Mais cela peut difficilement être considéré comme une condition constitutive du jugement. En d'autres termes, le contrôle rationnel sur nos jugements ne peut pas être constitué par le fait que soyons conscients de les effectuer, que ce contrôle soit réflexif ou non ${ }^{5}$.

Ce que McDowell veut dire est peut être différent, et c'est ce que suggère le fait qu'il parle "d'actualisation de capacités ». Il peut vouloir dire que c'est la possibilité d'exercer la capacité au jugement critique qui nous rend libres dans le domaine de la pensée. Cela peut se produire en particulier quand on relève un conflit entre le jugement et les normes de rationalité. Mais comment cette capacité peut-elle être déjà présente dans la perception à travers le fait qu'elle est conceptuelle si l'on doit la comprendre comme un développement supplémentaire de la capacité de juger à travers une pensée consciente?

Une description plus plausible de notre capacité à juger pourrait être la suivante. Nous avons des croyances de premier ordre, qui sont rationnelles ou pas. Nous n'avons aucun contrôle sur elles. Elles s'imposent seulement à nous. Pas plus que nous n'avons de contrôle sur les normes de rationalité. Nous ne devenons pas rationnels du seul fait que nous sommes conscients de nos croyances et des normes qui les régissent. Sans doute, des conflits peuvent

4. Evans, 1982; Moran, 2000; Shah, 2003; Shah et Velleman, 2005.

5. Cf. Owens, 2000; Engel, 2002a; Engel, 2009. 
se produire entre nos croyances du premier ordre et ce que nous croyons que nous devrions croire (ou ne pas croire). Et dans ces cas nous pouvons essayer de changer nos croyances. Mais on ne fait pas cela parce que l'on est conscient de ses normes et de ses croyances. On le fait parce qu'on a à se conformer à ces normes, et qu'on y est forcé.

Cette version faible de l'idée de contrôle rationnel sur nos jugements semble plus plausible que l'image néo-kantienne de l'autonomie et de la liberté de jugement suggérée par la première citation de McDowell. Selon cette version, la conscience que nous avons de nos croyances et des normes rationnelles est une précondition du contrôle rationnel; si nous avons cette conscience, nous sommes en position d'accepter certaines croyances et d'en rejeter d'autres. Mais cette version n'implique pas que le contrôle s'effectue à travers cette conscience de nos croyances et de leurs normes. Elle dit qu'il est une condition nécessaire, mais pas suffisante pour le contrôle rationnel. Il ne fait pas de doute que, pour pouvoir maintenir une certaine croyance, s'y tenir, ou bien la rejeter, il faut en être conscient. Et pour l'évaluer, je dois être conscient des normes qui la gouvernent. Il est tout à fait compatible avec la version faible du contrôle rationnel de nier qu'il y ait un contrôle actif sur la pensée. Le rôle de l'action dans la pensée est au mieux indirect. Il est préparatoire, et non pas constitutif de la pensée. En ce sens il n'y a pas de raison de se démarquer du slogan empiriste humien traditionnel selon lequel « la croyance appartient à la partie sensitive plutôt que cognititive de notre nature».

Il se peut que j'aie mal compris McDowell sur ces points, mais quoi qu'il en soit, j'aimerais en savoir plus que ce que disent ces quelques passages un peu énigmatiques sur la liberté de croire.

\section{Références}

Blackburn, S. «Practical Tortoise Raising ", Mind 104, 416, 695-711.

Brandom, R. Making it Explicit, Harvard, Harvard University Press, 1994.

Burge T. «Reason and the First Person », in C. Mc Donald et C. Wright, Knowing our Own Minds, Oxford University Press, 1995.

Engel, P. «L'espace des raisons est-il sans limites? " in Un siècle de philosophie, Paris, Gallimard, 2000, pp. 231-276.

- "The False Modesty of the Identity Theory of Truth", International Journal of Philosophical Studies, 9, 4, 2001, 441-458.

—. "Free Believers", vol. XXV, André Leclerc, Giovanni da Silva de Queiroz et Michael B. Wrigley (dir.), 2002, pp. 155-175.

__. «Le contenu de la perception est-il conceptuel ? » in J. Bouveresse et J. J. Rosat (dir.), Philosophies de la perception, phénoménologie, grammaire et sciences cognitives, Paris, Odile Jacob, 2003, 244-261.

—. "Epistemic Responsibility without Epistemic Agency ", Philosophical Explorations, vol. 12, $\mathrm{n}^{\circ}$ 2, juin 2009, 205-219.

Evans, G. The Varieties of Reference, Oxford, Oxford University Press, 1982.

Hieronymi, P. «Controlling attitudes », Pacific Philosophical Quarterly, 87 (1), 2006, $45-74$. 
McDowell J. "Having the World in View», Journal of Philosophy, 95, 1998, pp. 431-450.

—. 2002. "The True Modesty of the Identity Theory of Truth", International Journal of Philosophical Studies 13, 2004, 1, 82- 89.

Moran, R. Authority and Estrangement, Princeton, Princeton University Press.

Owens, D. Reason without Freedom, London, Routledge, 2000.

Shah N. "How Truth Regulates Belief», Philosophical Review, 112, 4, 2003, 447.

Shah N. et D. Velleman. «Doxastic Deliberation", Philosophical Review, 114, 4, 2005, 497. 